'Tbilisi State Medical University, Tbilisi,Georgia

${ }^{2}$ lashvili Central Children Hospital, Tbilisi, Georgia

${ }^{3 \prime}$ Test-Medical House" Diagnostic Centre, Tbilisi, Georgia

\title{
Prognostic value of TH1/TH2 cytokines in infants with wheezing in a three year follow-up study
}

The authors declare no financial disclosure

\begin{abstract}
Introduction: The immune system is known to play a key role in the development of viral-induced wheeze, but the pattern of cytokine response is not clear. The aim of the study was to determine whether plasma cytokine levels during the acute wheezing illness in infants are associated with the subsequent development of persistent recurrent wheezing (PRW).

Material and methods: 43 infants admitted to lashvili Central Children Hospital, Tbilisi, Georgia, were selected. The concentrations of IFN- $\gamma$, TNF- $\alpha$ and IL- 6 were determined by enzyme-linked immunosorbent assay (ELISA). Total serum IgE level was also determined. IgM and IgG antibodies to RSV, Chlamydophila pneumonia and Mycoplasma pneumonia were tested by ELISA. All children were followed-up during 3 years period.

Results: There was no significant association with PRW and atopy in the infant or parental atopy, low maternal age, low birth weight, serum level of IL-6, serum level of IFN- $\gamma$, serum level of total IgE and the particular pathogen. There was a tendency for the association with male sex, bottle feeding and household cigarette smoking, although the relation was not significant. There was just TNF- $\alpha$ which was strongly associated with the risk of PRW after three years of follow up.

Conclusion: We have identified TNF- $\alpha$, as a biomarker, which can be detected in children under 3 years of age and may be useful in the prediction of development of persistence of wheezing later. To further evaluate the usefulness of this and other markers and establish cutoff levels for prediction and use in clinical practice, a larger prospective follow-up study is needed.
\end{abstract}

Key words: cytokines, biomarker, pediatric asthma, wheezing

Pneumonol Alergol Pol 2016; 84: 145-150

\section{Introduction}

Wheezing in infancy is very common, about $1 / 3$ of infants experiencing at least one wheezing episode by their third birthday, and half of them by their sixth birthday. However, not all infants who experience wheezing continue to wheeze. Approximately one half of those wheezing before 3 year of age are asymptomatic by age of 6 . The long-term prognosis of early wheezing is, in most cases, good. The vast majority of early wheezers either wheezes no more after the age of 3 years (early remittent wheezers) or only in some years (early intermittent wheezers) up to the age of $13 \mathrm{yrs}$. Only a very small fraction of early wheezers (early persistent wheezers) keeps on wheezing every single year up to the age of 13 years [1,2]. At present, it is not understood why wheezing illness persists in some children, when in most it resolves spontaneously.

It is apparent that infants and preschool children that wheeze with viral infections may have persistent symptoms and progress to develop asthma by school age. A major pediatric issue is how to identify the infants whose wheezing represents transient reactions in response to different 
respiratory infections, and to distinguish those children from those whose wheezing represents the initial phase of persistent asthma. Findings to date strongly suggest that the impact of infant viral infections on the maturing of immune system and developing lung, that subsequently result in an asthma phenotype occur during a critical susceptibility period, and in a genetically susceptible host. There are currently no therapeutic strategies that allow prevention of asthma following early life viral respiratory infections in high-risk children, thus a focus on understanding the mechanisms of progression from viral wheezing in infants and preschool children to asthma development are urgently needed [3].

The immune response, in particular $\mathrm{T}$ cell responses are known to play a key role in the development of viral-induced wheeze. However, the pattern of cytokine response in wheezing infants is not clear. Th1 lymphocyte cytokine production (interferon- $\gamma$ and interleukin-2) is responsible for the cell-mediated immune response. Th2 lymphocyte cytokine production (interleukin-4 and interleukin-6) stimulates antibody production, especially IgE response, also enhancing eosinophil production and activity. Some studies are indicating that either Th1 or Th2 responses may predominate in infants with acute bronchiolitis, other studies, however, have reported that there is no specific Th1 or Th2 pattern of response in infants with this disease $[4,5]$. One recent study demonstrated, that persistently increased production of TNF- $\alpha$ in response to LPS stimulation of blood mononuclear cells at birth and at 3 months (not later) is predictive of the development of childhood asthma [6]. We undertook this study in order to determine whether plasma cytokine levels during the acute wheezing illness in infants are associated with the subsequent development of persistent recurrent wheezing.

\section{Material and methods}

We conducted an observational prospective cohort study in 2009-2012. Infants between three month and three years of age admitted to Iashvili Central Children Hospital, Tbilisi, Georgia, with acute wheezing were selected for the study.

The Ethics Committee at Tbilisi State Medical University approved the study and informed consent was obtained from the parents prior to enrolment.

Inclusion criteria were patients aged $>3$ month and $<3$ years with a respiratory symptom duration of $<3$ days. Respiratory symptoms inc- lude prodromal rhinorrhea and cough, followed by at least 2 of the following signs: tachypnea, wheezing, rales, use of accessory muscles, and/or nasal flaring. Additional inclusion criteria included first episode of wheezing or shortness of breath, and informed consent.

Infants with extended crepitations on auscultation and bronchopneumonic infiltration in postero-anterior lung X-ray without sibilant rhonchi and prolongation in expiration were defined as pneumonia and were excluded from the study. Infants which were administered antihistamines and corticosteroids in any form during current illness were also excluded from the study.

Baseline observations including temperature, oxygen saturation, respiratory and pulse rates were recorded at the time of admission and daily thereafter in all participants. Total leukocyte counts with differential and C-reactive protein (CRP) levels were studied in all patients. Chest radiographs of all cases were evaluated by a pediatric radiologist.

Samples of blood sera were obtained within the first $48 \mathrm{~h}$ of admission. The concentrations of IFN- $\gamma$, TNF- $\alpha$ (TH1) and IL-6 (TH2) were determined by enzyme-linked immunosorbent assay (ELISA), with a detection limit of 25.0, 6.0 and $30.0 \mathrm{pg} / \mathrm{ml}$, respectively. Results were considered to be negative when cytokine concentrations were below the detection limit. Total serum IgE level was also determined. Acute and convalescent serum samples were tested by ELISA for IgM and IgG antibodies to Respiratory Syncytial Virus, Chlamydophila pneumonia and Mycoplasma pneumonia (manufacturer Vircell Microbiologists, Spain). Positive results were defined by a significant antibody response in specific IgM or a 4 -fold increase in IgG titer in paired serum samples. A positive infection was defined either in a single test or in paired sera taken $2-3$ weeks apart.

Occurrences of subsequent wheezing were obtained from parental responses on questionnaires and/or during physicians' assessment either in inpatient or outpatients settings. All cases of hospital admission were recorded. Date were collected 1,2 and 3 years later after enrolment in the study. Those children who had 3 or more episodes of wheezing each year of follow up were considered as a persistent recurrent wheezers [7].

The results have been analyzed by the SPSS Statistics versions 16.0 (IBM Corp, Amonk, NY). All data were entered onto a dedicated database and checked for errors. Thirteen variables were chosen as being of special interest: (1) atopic dermatitis in the infant; (2) low maternal age 
(less than 20 years); (3) low birth weight; (4) parental atopy; (5) household cigarette smoking; (6) exclusive breastfeeding up to 6 month of age; (7) sex; (8) serum level of IL-6; (9) serum level of IFN-g; (10) serum level TNF- $\alpha$; (11) serum level of total IgE; (12) RSV etiology of wheezing; (13) atypical etiology (Chlamydophila pneumonia, Mycoplasma pneumonia) of wheezing. Each variable was entered separately into univariate models. Estimated logistic regression coefficients, standard errors, odds ratios, and p-value from the score test statistic on the significance for each variable's inclusion into each univariate model were calculated. $\mathrm{P}<0.05$ has been considered as significant difference. Frequencies were computed without excluding missing answers, which were therefore counted as negative. Missing answers were always lower than 5\%. Associations between several variables of wheezing children were assessed. Results are presented as odds ratios (ORs) and 95\% confidence intervals (95\% CI).

Patients were grouped according to pathogens in three groups: in group I patients with atypical pathogens - Chlamydophila pneumoniae and Mycoplasma pneumoniae were included; in group II there were patients with $R S V$; in group III there were patients with mixed-infections with Chlamydophila pneumoniae, Mycoplasma pneumoniae and Respiratory syncytial virus.

\section{Results}

Of 47 children enrolled in the cohort, 43 (91\%) had at least 1 follow-up contact during the three year period and were included in the study.

\section{Subject characteristics}

The study group consisted of 43 patients with lower respiratory tract infection with wheezing. Distribution of the patients of this group according their age is shown in Figure 1.

Median age of the patients was 13.8 months, twenty-seven patients (63\%) were boys. Nine patients $(20.9 \%)$ were born prematurely. Thirteen patients $(30.2 \%)$ were on formula feeding up to 6 month of age. Eighteen patients (41.8\%) had a positive family history of atopy. Thirty patients (69.7\%) were under environmental tobacco exposure (smoking mother or father). None of the patients received ribavirin or systemic anti -inflammatory agents, including corticosteroids before enrolment.

In the control group, 6 children were boys $(60 \%)$, the median age was 14.8 months and 2 controls (20\%) were born prematurely. Five

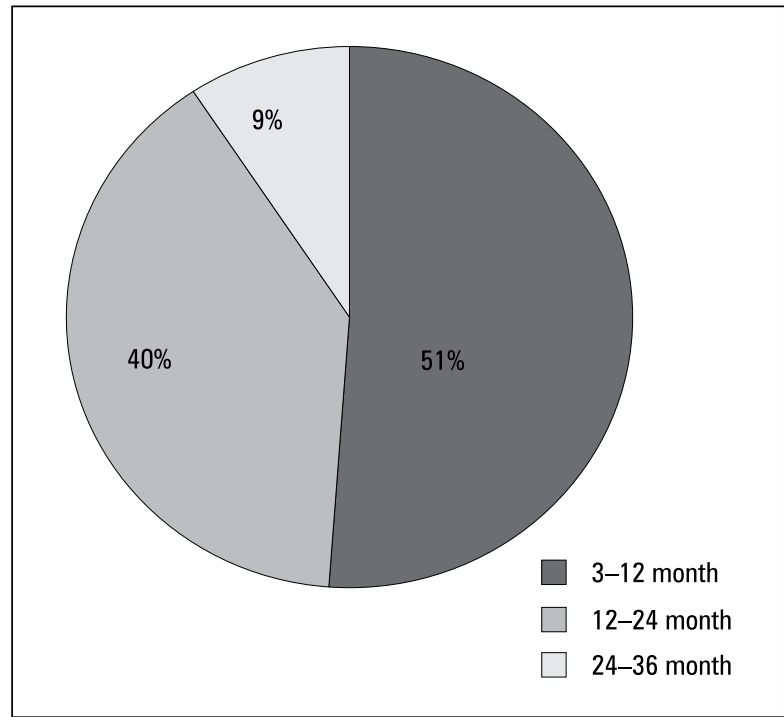

Figure 1. Patients distribution by age (\%)

patients $(50 \%)$ were on formula feeding up to 6 month of age. Two patients (20\%) had a positive family history of atopy. Six patients (60\%) were under environmental tobacco exposure (smoking mother or father).

Out of 43 patients etiological diagnosis were established in 32 patients (74.4\%). They were grouped according to pathogens in three groups:

Group I involved 13 patients, who had either Chlamydophila pneumoniae or Mycoplasma pneumoniae or their co infections. In this group the median age was 13.7 month (age range from 4 to 36 months).

Group II involved 10 patients with $R S V$, their median age was 12.6 month (age range from 4 to 30 months).

Group III involved 9 patients, who had co infections of Chlamydophila pneumoniae, Mycoplasma pneumoniae and RSV. Their median age was 10.2 month (age range from 4 to 18 months). Co-infection with two pathogens were detected in 7 (18.9\%) samples, three pathogens in 2 (4.6\%) sample.

Out of 43 children no wheezing episodes after one years follow-up had two (4.6\%) children, after two year nine $(20.9 \%)$ and after three years fifteen (34.8\%) children. Three and more episodes after one year follow-up had twenty (46.5\%) children, after two years - nineteen (44.1\%) and after three years - sixteen (37.2\%) children (Fig. 2).

In children with persistent recurrent wheezing there was a tendency for the association with male sex, bottle feeding and household cigarette smoking, although the relation was not significant. 


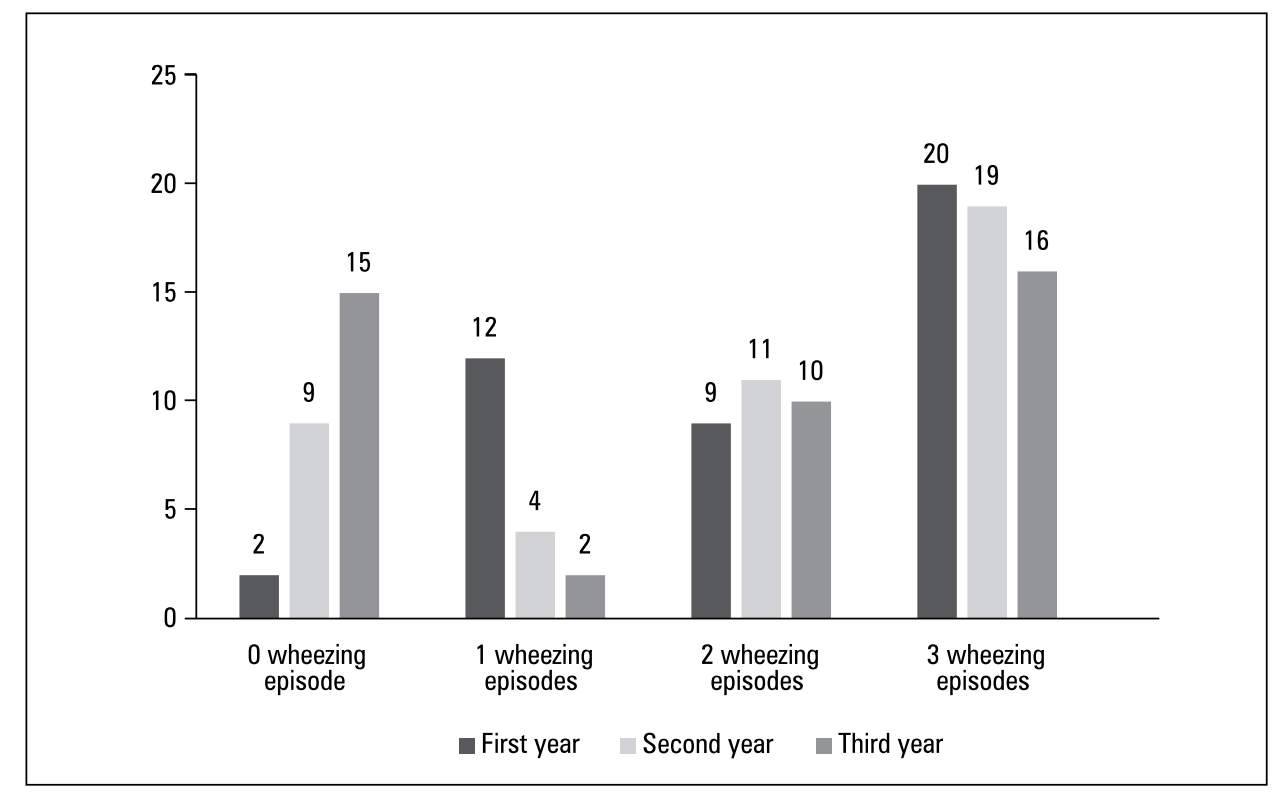

Figure 2. Wheezing episodes during three years of follow up period

Table 1. Independent odds ratios and confidence intervals for the effects of each variables on the risk recurrent wheezing for the three year of follow up

\begin{tabular}{lccc}
\hline Factor & OR & $\mathbf{9 5 \%}$ CI & p-value \\
\hline Atopic dermatitis in the infant & 1.591 & $0.395-6.407$ & 0.512 \\
Parental atopy & 2.571 & $0.721-9.167$ & 0.141 \\
Household cigarette smoking & 0.926 & $0.242-0.543$ & 0.911 \\
Exclusive breastfeeding up to 6 month of age & 0.667 & $0.167-2.666$ & 0.565 \\
Low maternal age & 2.614 & $0.584-11.694$ & 0.200 \\
Low birth weight & 1.094 & $0.791-1.502$ & 0.611 \\
Sex & 1.512 & $0.410-5.586$ & 0.534 \\
IL-6 & 2.083 & $0.588-7.383$ & 0.252 \\
IFN- $\gamma$ & 0.500 & $0.080-2.841$ & 0.428 \\
TNF- $\alpha$ & 8.75 & $1.656-46.237$ & $<0.005^{*}$ \\
Total lgE & 1.146 & $0.322-4.081$ & 0.834 \\
RSV etiology & 1.167 & $0.274-4.976$ & 0.835 \\
CPN etiology & 2.614 & $0.584-11.694$ & 0.200 \\
MPN etiology & 0.659 & $0.144-3.021$ & 0.590 \\
\hline
\end{tabular}

IL-6 — interleukin-6; IFN- $\gamma$ — interferon gamma; TNF- $\alpha$ — tumor necrosis factor-alpha; IgE — immunoglobulin E; RSV — respiratory syncytial virus; CPN — Chlamydophila pneumoniae; MPN - Mycoplasma pneumoniae; ${ }^{*} \mathrm{p}<0.05$ considered significant

There was no significant association with atopy in the infant or parental atopy, low maternal age, low birth weight, serum level of IL-6, serum level of IFN- $\gamma$ and serum level of total IgE.

Persistent recurrent wheezing after three years of follow up also was not associated with the particular pathogens: there was no difference between RSV, Chlamydophila pneumonia and Mycoplasma pneumonia etiology of wheezing.
There was just TNF- $\alpha$ which was strongly associated with the risk of persistent recurrent wheezing after three years of follow up (OR = 8.75; 95\% CI 1.656-46.237, $\mathrm{p}<0.05$ ). TNF- $\alpha$ was not associated with specific etiology of infection.

Independent odds ratios and confidence intervals for the effects of each variables on the risk of recurrent wheezing for the three years of follow up is shown in Table 1. 


\section{Discussion}

Evidence that early intervention with anti -inflammatory agents leads to an amelioration of future disease is increasing, and the demand for rational treatment strategies for wheezing in infancy is growing [8, 9]. It is clear, that it would be pointless to treat all wheezy infants as in most cases the condition will resolve spontaneously [10]. Therefore, an identifiable marker that would indicate whether an infant is likely to develop genuine asthma would be the most useful. Thus, the accurate selection of subjects in whom the benefits of early intervention outweigh the disadvantages has become a most important issue $[11,12]$. The identification of determinants for wheeze in early childhood has been the subject of several investigations [2, 3, 5, 13-18]. But, to the best of the author's knowledge, this is the first study to quantify different factors, such as wheezing etiology (included atypical pathogens), demography, family history, household environment, feeding and pro- and anti-inflammatory cytokines level in early life to identify those markers, which might be predictive for the persistence of wheezing later.

Using univariate logistic regression, we found that children were more likely to experience persistent symptoms if, at presentation, they have elevated levels of TNF- $\alpha$. There was a tendency for the association of recurrent wheezing with male sex, bottle feeding and household cigarette smoking, although the relation was not significant. There was no significant association with atopy in infant or parental atopy, low maternal age, low birth weight, serum level of IL-6, serum level of IFN- $\gamma$ and serum level of total IgE.

In the majority of papers no significant correlations were observed between the level of IL-6, IFN- $\gamma$ and recurrent wheezing [4, 6, 9], but in other studies IFN- $\gamma$ level was significantly higher among patients with recurrent wheezing than among those without recurrent wheezing $[8,19]$.

In our study we found a direct correlation of TNF- $\alpha$ level with the development of recurrent wheezing later in life. The same results were showed in other studies, but not at the same age in patients under 3 months $[6,20]$. So, in spite of that our study is not the first study to suggest that TNF- $\alpha$ is related to asthma, it is the first study to show prospectively a relation of early-life TNF- $\alpha$ to persistency of wheezing and asthma later.

These results suggest that TNF- $\alpha$ could be considered as molecular predictor for recurrent wheezing. Only TNF- $\alpha$ (and not IL-6 or
IFN- $\gamma$ ) showed a significant relation to recurrent wheezing by logistic regression. This specificity raises the possibility that TNF- $\alpha$ may have a preferential or even unique biologic role in asthma development. Although asthma is most often not diagnosed until age 6 , this does not mean that the process is not initiated much earlier in life. It is also possible that the relation of TNF- $\alpha$ to asthma does not have a direct biologic basis and simply serves as a biomarker [6].

We have not found significant association between birth weight and recurrent wheezing. Sonnenschein-van der Voort et al. [21] performed an individual participant data meta-analysis of 147,252 children in 31 birth cohort studies to determine the associations of birth and infant growth characteristics with the risks of preschool wheezing and school-age asthma. Compared with children with a normal birth weight, those with a low birth weight (less than $2500 \mathrm{~g}$ ) had an increased risk of preschool wheezing and school-age asthma, but the associations of lower birth weight with childhood asthma were largely explained by gestational age at birth. Tadaki $\mathrm{H}$. et al. [22] investigated cord blood cytokines in a prospective birth cohort, intensively monitored for wheezy infant outcome at $1 \mathrm{yr}$. It was found out that risk of wheezing was related to birth weight, as well as cesarean section, and maternal eczema. Jaakkola et al. [23] performed a meta-analysis on the associations of preterm birth with asthma based on 19 published cohort, case control, and cross-sectional studies. They observed that preterm born children, which were defined as those born before 37 weeks of gestation, had an increased risk of asthma between 1 and 24 years of age, with a similar effect estimate as observed in our group of 5 - to 10 -year-olds. They did not assess associations of birth weight with asthma outcomes.

Although follow-up of this group continues and the whole group will be reevaluated clinically at 6 years of age, the length of follow-up reported in our study is 3 years. If, at the beginning of wheezing in children, it could be predicted that disease would continue for 3 years, the majority of physicians would regard more frequent follow-up and prophylactic treatment as being appropriate.

Although an exact diagnosis of asthma will not be possible until 6 year of age or older, there is a need to identify which children have higher risk of asthma development that early intervention studies can be planned. The identification of the high persistent production of this cytokine as a biomarker could provide a major advance in therapeutic design of prevention strategies. 


\section{Conclusion}

In conclusion, we have identified TNF- $\alpha$, as a biomarker, which can be detected in age under 3 years and may be useful in the prediction of persistence of wheezing later. To further evaluate the usefulness of this and other markers and establish cutoff levels for prediction and use in clinical practice, a larger prospective follow-up study is needed.

\section{Conflict of interest}

The authors declare no conflict of interest.

\section{References:}

1. Martinez F, Wright A, Taussig L et al. Asthma and wheezing in the first six years of life. N. Engl. J. Med. 1995; 332: 133-138. doi: 10.1056/NEJM199501193320301.

2. Matricardi P, Illi S, Gruber C, Keil T et al. Wheezing in childhood: incidence, longitudinal patterns and factors predicting persistence. Eur Respir J 2008; 32: 585-592. doi: 10.1183/09031936.00066307.

3. Saglani S. Viral infections and the development of asthma in children. Ther Adv Infect Dis 2013; 1: 139-150. doi: 10.1177/ /2049936113497202.

4. Pitrez P, Machado D, Andrade F, Camozzato C, Stein R. Th-1 and Th-2 cytokine production in infants with virus-associated wheezing. Braz J Med Biol Res 2005; 38: 51-54. doi. org/10.1590/S0100-879X2005000100008.

5. Sarria E, Mattiello R, Yao W et al. Atopy, cytokine production, and airway reactivity as predictors of pre-school asthma and airway responsiveness. Pediatr Pulmonol 2014; 49: 132-139. doi:10.1002/ppul.22784.

6. Halonen M, Lohman C, Stern D et al. Perinatal tumor necrosis factor- $\alpha$ production, influenced by maternal pregnancy weight gain, predicts childhood asthma. Am J Respir Crit Care Med 2013; 188: 35-41. doi: 10.1164/rccm.201207-1265OC.

7. Castro-Rodriguez JA. The Asthma Predictive Index: early diagnosis of asthma. Curr Opin Allergy Clin Immunol 2011; 11: 157-161.doi: 10.1097/ACI.0b013e3283464c4a.

8. Sugai K, Kimura H, Miyaji Y et al. MIP-1 $\alpha$ level in nasopharyngeal aspirates at the first wheezing episode predicts recurrent wheezing. J Allergy Clin Immunol 2015; 19: 01256-7. doi: 10.1016/j.jaci.2015.08.032.

9. Takeyama A, Hashimoto K, Sato $M$ et al. Clinical and epidemiologic factors related to subsequent wheezing after virus-induced lower respiratory tract infections in hospitalized pediatric patients younger than 3 years. Eur J Pediatr 2014; 173: 959-966. doi: 10.1007/s00431-014-2277-7.

10. Bilolikar H, Nam A, Rosenthal M, Davies J, Henderson D. Balfour-Lynn I. Tumour necrosis factor gene polymorphisms and childhood wheezing. Eur Respir J 2005; 26: 637-646. doi: 10.1 183/09031936.05.00071104.

11. Clough J, Keeping K, Edwards L, Freeman W, Warner JA, Warner JO. Can we predict which wheezy infants will continue to wheeze? Am J Respir Crit Care Med 1999; 160: 1473-1480. doi: 10.1164/ajrccm.160.5.9807019.

12. Brown PM, Schneeberger DL, Piedimonte G. Biomarkers of respiratory syncytial virus (RSV) infection: specific neutrophil and cytokine levels provide increased accuracy in predicting disease severity. Paediatr Respir Rev 2015; 16: 232-240. doi: 10.1016/j.prrv.2015.05.005.

13. Bertrand P, Lay MK, Piedimonte G et al. Elevated IL-3 and IL-12p40 levels in the lower airway of infants with RSV-induced bronchiolitis correlate with recurrent wheezing. Cytokine. 2015; 76: 417-423. doi: 10.1016/j.cyto.2015.07.017.

14. Hollams E, de Klerk N, Holt P, Sly P. Persistent effects of maternal smoking during pregnancy on lung function and asthma in adolescents. Am J Respir Crit Care Med 2014; 189: 401-407. doi: 10.1164/rccm.201302-0323OC.

15. Message S, Laza-Stanca V, Mallia P et al. Rhinovirus-induced lower respiratory illness is increased in asthma and related to virus load and Th1/2 cytokine and IL-10 production. Proc Natl Acad Sci USA 2008; 105: 13562-13567. doi: 10.1073/ pnas.0804181105.

16. Bont L, Heijnen C, Kavelaars A et al. Monocyte IL-10 production during respiratory syncytial virus bronchiolitis is associated with recurrent wheezing in a one-year follow-up study. Am J Respir Crit Care Med 2000; 161: 1518-1523. doi: 10.1164/ ajrccm.161.5.9904078.

17. Lieke L, Reubsaet M, Meerding J et al. Plasma chemokines in early wheezers predict the development of allergic asthma. Am J Respir Crit Care Med 2013; 188: 1039-1040. doi: 10.1164/ rccm.201212-2330LE.

18. Pala P, Bjarnason R, Sigurbergsson F, Metcalfe C, Sigurs N, Openshaw P. Enhanced IL 4 responses in children with a history of respiratory syncytial virus bronchiolitis in infancy. Eur Respir J 2002; 20: 376-382. doi: 10.1183/09031936.02.00249902.

19. Jartti T, Paul-Anttila M, Lehtinen P et al. Systemic T-helper and T-regulatory cell type cytokine responses in rhinovirus vs. respiratory syncytial virus induced early wheezing: an observational study. Respir Res 2009; 10: 85. doi: 10.1186/14659921-10-85.

20. McNamara P, Flanagan B, Selbyz A, Hart C, Smyth R. Proand anti-inflammatory responses in respiratory syncytial virus bronchiolitis. Eur Respir J 2004; 23: 106-112. doi: 10.1183/09 031936.03.00048103.

21. Sonnenschein-van der Voort A, Arends L, de Jongste J at al. Preterm birth, infant weight gain, and childhood asthma risk: a meta-analysis of 147,000 European children. J Allergy Clin Immunol 2014; 133: 1317-1329. doi: 10.1016/j. jaci.2013.12.1082

22. Tadaki H, Arakawa H, Sugiyama M et al. Association of cord blood cytokine levels with wheezy infants in the first year of life. Pediatr Allergy Immunol 2009; 20: 227-233. doi: 10.1111/j.1399-3038.2008.00783.x.

23. Jaakkola JJ, Ahmed P, Ieromnimon A et al. Preterm delivery and asthma: a systematic review and meta-analysis. J Allergy Clin Immunol 2006; 118: 823-830. doi: http://dx.doi.org/10.1016/j. jaci.2006.06.043. 